\title{
Statistical Analysis of Simulations of Coarsening Droplets Coating a Hydrophobic Surface
}

\author{
Jeremy Semko
}

May 27, 2010

\begin{abstract}
Thin layers of slow-moving, viscous fluids coating hydrophobic surfaces are shaped by the competing forces of disjoining pressure and surface tension. These forces form the fluid layer into an array of discrete droplets connected by an ultra thin layer. However, the droplet array is unstable, and the droplets will interact with one another. To determine the structure and properties of steady droplets, we use the Reynolds' PDE in one dimension and phase-plane methods. We can then analyze the unstable droplet system by utilizing paired ODEs. Numerical solutions show how the droplets interact to produce movement and mass exchange, giving rise to coarsening events which reduce the number of droplets in the system. These events occur when a droplet collapses into the ultra thin layer or when two droplets collide, and thus, merge. Using numerical simulations and statistical analysis of their results, we aim to gain a better understanding of the dynamics of this system including the factors that influence coarsening events such as parameters and initial conditions.
\end{abstract}

\section{Introduction}

In past studies $[10,11]$, viscous, slow-moving fluids which coat hydrophobic solid surfaces have shown evidence of instability characterized by a nearly uniform layer breaking up into an array of droplets connected by an ultra thin layer. This particular phenomenon is called dewetting, and it occurs in many fluid dynamics systems. For example, experiments on different polymer solutions $[2,6]$ have attempted to pin down the peculiar nature of dewetting. More simply however, this behavior is also exhibited in everyday materials such as printing ink, paint, and lubricant. Clearly this action can have negative effects due to the complicated and somewhat unpredictable patterns it creates. For this reason, the changing shape of these fluid layer has been the subject of research, yet many aspects are not yet fully understood.

Additionally, dewetting is not the final instability that many of these systems experience. For materials that do not evaporate (such as lubricant), the droplets, which are connected by a thin layer of fluid, are still unstable and will continue to interact with one another by means of fluid flux. These interactions can create movement and 
mass exchange that occur over long time scales. Moreover, these droplets can also experience certain critical "events". In particular, a droplet can become so small that it collapses into the ultra thin layer, or two droplets can get close enough that they collide and merge into a larger drop. These actions are called coarsening, and lead to a system which contains a smaller number of larger drops (since mass is conserved).

We will begin by using a phase-plane system to show how the shape of droplets is derived from equations of fluid dynamics. Next, we describe the differential equations that will govern the dynamics of the system of droplets and see how we will be able to simulate this system, keeping in mind the changes we must make when certain "events" occur. After this foundation is built, we will aim to explain the factors contributing to the evolution of the number of droplets throughout a simulation. Lastly, we narrow this focus to specifically how these factors contribute to either collapses or collisions of droplets.

\section{Formulation of the thin film problem}

We describe dynamics occurring for fluid systems that all share a number of characteristics. The fluids are thick and very viscous and hence will have strong dissipative friction. They are also slow-moving, which we will understand to mean they have a very low Reynolds number. They are also non-volatile, so they do not evaporate. In addition, the fluid will be in a hydrophobic container with no-flux boundary conditions.

We have mentioned that physical experiments have shown that a nearly uniform layer of a thin film will break up due to instabilities $[2,6]$. Our first step will be to write the governing equations that model this dewetting.

The dynamics of viscous fluids are given by the Navier-Stokes partial differential equations. The Reynolds number of a fluid is a parameter in the Navier-Stokes systems that describes the speed versus the dissipation of a fluid. Because we are modeling very slow moving fluids, this parameter is close to zero, which reduces the PDEs to the Stokes equations [1]. Further reducing the problem, we will be analyzing thinfilms in one dimension. This leads to the Reynolds equation in one dimension, which is reduced from the Stokes equations and describes the evolution and conservation of mass of thin-films $[3,7-9]$. This is

$$
\frac{\partial h}{\partial t}+\frac{\partial}{\partial x}\left(h^{3} \frac{\partial p}{\partial x}\right)=0
$$

where $h$ is the height or thickness of the fluid layer, $t$ is the time, $p$ is the pressure, and $x$ is the position in the layer with $0 \leq x \leq L$. Also $h^{3} \frac{\partial p}{\partial x} \equiv J$ is defined as the flux.

The forces acting on the fluid are represented by contributions to the pressure; these are the surface tension of the fluid and intermolecular forces, or what is also called the disjoining pressure. The former depends on the curvature of the surface and the latter depends on the forces between the particles of the fluid with one another and between the fluid molecules and the hydrophobic solid. We denote the disjoining pressure as $\Pi(h)$. Because $\left|h_{x}\right| \ll 1$ for thin-films, curvature in two dimensions, where our dimensions are position and height can be reduced to a one-term approximation, 


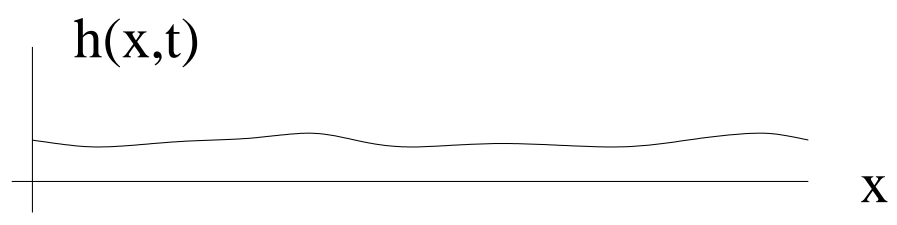

Figure 1: Graph of typical thin-film fluid.

or

$$
\kappa=-\frac{h_{x x}}{\left(1+h_{x}^{2}\right)^{3 / 2}} \approx-\frac{\partial^{2} h}{\partial x^{2}}
$$

The fluid's pressure can be written as a sum of these two terms $[10,11]$. Thus, we write pressure as

$$
p=\Pi(h)-\frac{\partial^{2} h}{\partial x^{2}}
$$

One particular equation for the $\Pi(h)$ term that describes a non-wetting or partially wetting fluid on a water repellent coated solid is (from $[4,11]$ )

$$
\Pi(h)=\frac{\delta^{2}}{h^{3}}\left(1-\frac{\delta}{h}\right)
$$

where $\delta$ is the height of the ultra thin layer that coats the region between separated droplets.

\subsection{Droplet Solutions to the Steady-State Equations}

We now derive the properties of steady droplet solutions existing in an idealized infinite domain. If the fluid is steady then (1) reduces to

$$
\frac{\partial}{\partial x}\left(h^{3} \frac{\partial p}{\partial x}\right)=0
$$

on a fixed length domain with no-flux boundary conditions (so $J=0$ at the boundaries). In order for (5) to hold with $h>0$, the mass flux, $J=h^{3} \frac{\partial p}{\partial x}$ must be a constant with respect to $x$. However, $J=0$ on the boundary so the constant is 0 . Because $h \neq 0$, $\frac{\partial p}{\partial x}=0$. This implies that pressure is a constant.

Keeping in mind that a steady system implies pressure is constant, we can rewrite the steady form of (3) as

$$
\frac{d^{2} \bar{h}}{d x^{2}}=\Pi(\bar{h})-\bar{p}
$$

(where the bar indicates independence of time), or equivalently

$$
\frac{d^{2} \bar{h}}{d x^{2}}=\frac{\delta^{2}}{\bar{h}^{3}}-\frac{\delta^{3}}{\bar{h}^{4}}-\bar{p}
$$


If we write $\bar{z}=\frac{d \bar{h}}{d x}$ then the above equations reduce to the first order system

$$
\begin{aligned}
& \frac{d \bar{h}}{d x}=\bar{z} \\
& \frac{d \bar{z}}{d x}=\frac{\delta^{2}}{\bar{h}^{3}}-\frac{\delta^{3}}{\bar{h}^{4}}-\bar{p} .
\end{aligned}
$$

Solving for the $x$-independent solutions, we find that this system has two equilibrium points with $\bar{z}=0$. Using linear stability analysis, the solution near $\bar{h}=\delta$ is a saddle while the other equilibrium is a center point (see Figure 2(a)).

At this point, we know that at the maximum of a droplet, $\frac{d \bar{h}}{d x}=\bar{z}=0$. By using the ode45 solver in MATLAB, we can compute numerical solutions to this phase-plane system of differential equations by trying out various initial heights for a given $\bar{p}$ while keeping $\delta$ fixed. Using this shooting approach, we can obtain the solutions that are of interest to us. In particular, if $\bar{h}(0)$ is too large, the solution will yield an $\bar{h}$ that gets near the saddle point, but reverses direction, creating a solution with a diverging slope. This singularity is not exhibited by the drops and is, thus, not physically relevant. If $\bar{h}(0)$ is initially too small, then the solution will be periodic, orbiting around the center equilibrium point. This would indicate an infinite number of droplets along a domain. In between these two, there is a homoclinic solution that approaches the saddle point of the system along the asymptotes. This homoclinic solution to the system is the only solution that respects physical properties of an isolated individual drop in an infinite domain - having $\bar{h} \sim \delta$ and $\bar{z} \rightarrow 0$ as $|x| \rightarrow \infty$, see Figure 2(b). To deduce the homoclinic solution and the appropriate initial maximum height, we use a bisection search to find the unique value for the maximum of $\bar{h}$ for a given $\bar{p}$.

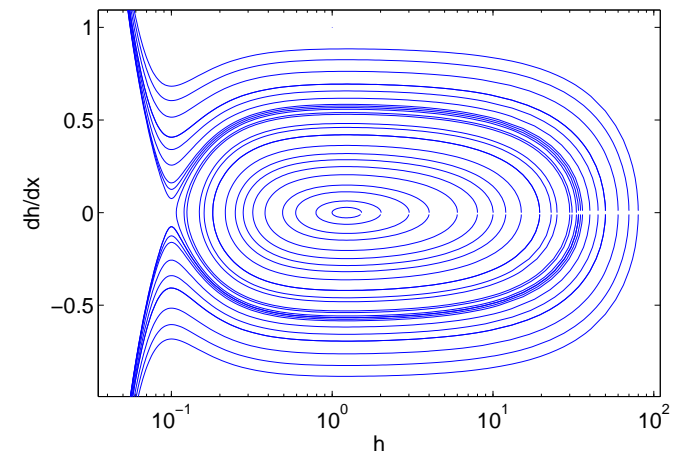

(a) Solutions to (8), the phase-plane system where $\delta=0.1$.

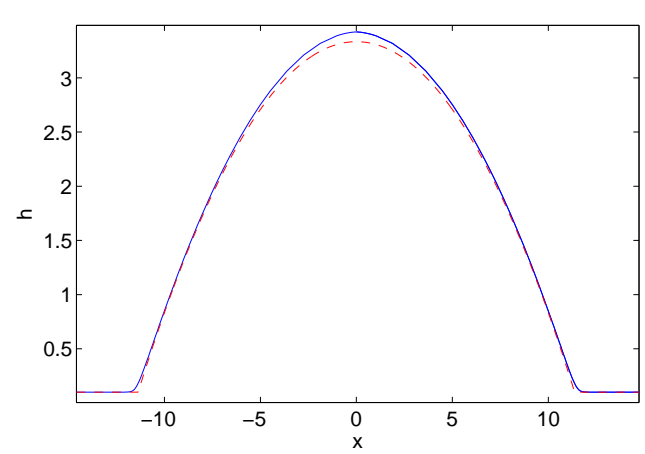

(b) Homoclinic solution (solid line) of the system vs. the approximate solution (10) $p=0.05$ (dotted line).

Figure 2: The homoclinic solution.

From (7), when $\delta$ is much smaller than $h$, the differential equation can be approximated by

$$
\frac{d^{2} \bar{h}}{d x^{2}}=-\bar{p}
$$


Therefore the droplet profile is approximated by a parabola. On the other hand, when $\bar{h}$ gets close to $\delta$, we can approximate $\bar{h}$ as just that, $\bar{h} \sim \delta$. By using the $\bar{h}(0)$ from the bisection search and solving (9), we can find a width $w$ of the drop. This also allows us to write an approximation of the profile of the drop as (see Figure 2(b))

$$
\bar{h} \sim \begin{cases}\frac{1}{2} \bar{p}\left(\bar{w}^{2}-x^{2}\right) & \text { if }|x|<\bar{w} \\ \bar{h}_{\text {min }}=\delta & \text { else. }\end{cases}
$$

We can vary the value of $\bar{p}$ when solving for the homoclinic solution to find good approximations for the width, in which case we observe the value of $x$ in the solution when $h$ gets close to $\delta$. We then can graphically observe the relationship between $\bar{p}$ and the width, as shown in figure 3(a). This relationship is very close to

$$
\bar{w} \sim \frac{A}{\bar{p}} .
$$

where $A$ is a constant determined by the form of the disjoining pressure. Clearly, if

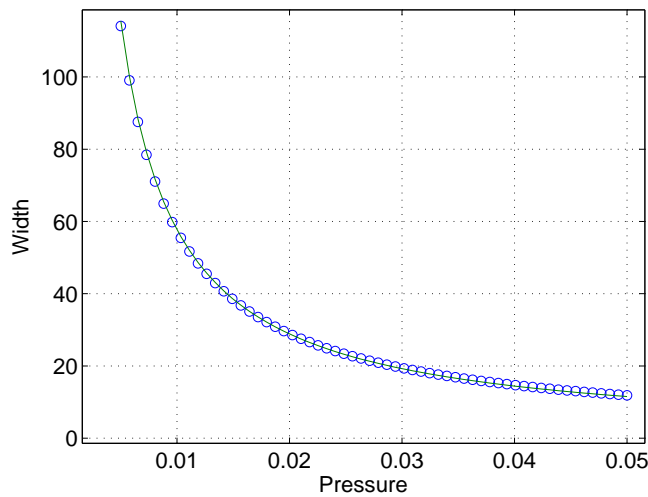

(a) Prediction by (11) (curve) compared with computed solutions of phase plane equation (data points) (8).

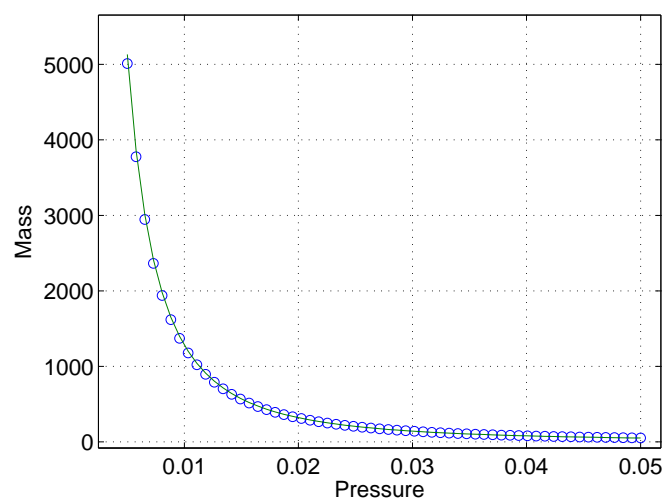

(b) Prediction by (12) (curve) versus computed solutions of phase plane equation (data points)(8).

Figure 3: Width and mass dependence on pressure.

density $=1$, then the mass $\bar{m}$ of a droplet is the area under the height function $\bar{h}$, which is

$$
\bar{m}=\int_{-w}^{w} \bar{h} d x \sim \frac{2 A^{3}}{3 \bar{p}^{2}}
$$

as shown in figure $3(\mathrm{~b})$. With equation (12) and (11), we can rewrite the mass as a function of pressure. In particular

$$
\bar{w}(\bar{m})=\sqrt{\frac{3 \bar{m}}{2 A}} .
$$




\section{Coarsening Dynamical System}

Now that we have the equations for some of the basic characteristics of droplets, given $X$, the position of a droplet, and $M$, the mass of a drop, we can write the height profile for each drop as

$$
h=\bar{h}(x-X, M) .
$$

Now we have built the basics of a droplet system. The next step is to determine how systems of interacting droplets change over time, and for this we will need equations that govern the evolution of positions and masses $X=X(t)$ and $M=M(t)$.

While the above steps show that a single droplet is steady and takes the approximate shape of parabola, in general, an array of multiple droplets does not form a global steady solution. In particular, the droplets may move horizontally or exchange mass, resulting in growth or shrinkage as described in the equations derived in $[10,11]$. From [5], a modified version of simplified ODEs, which have been reduced from (1), are, for droplets $i=1,2, \ldots, N$

$$
\begin{gathered}
\frac{d M_{i}}{d t}=\left(J_{i+1, i}-J_{i, i-1}\right) \\
\frac{d X_{i}}{d t}=C_{x}\left(M_{i}\right)\left(J_{i+1, i}+J_{i, i-1}\right)
\end{gathered}
$$

where $X_{i-1}<X_{i}, M_{i}$ is the mass of the $i^{t h}$ droplet, and $J_{i+1, i}$ is the flux $J$ between the $i+1^{t h}$ and $i^{t h}$ droplet. Here $C_{x}\left(M_{i}\right)$ is a coefficient that depends on a droplet's mass and, from [5], is approximated as inversely proportional to $\ln \left(M_{\delta} / M_{i}\right)$, and $M_{\delta}$ is the limit of the mass of a droplet whose height approaches the height of the ultra thin layer. Normally $C_{x}\left(M_{i}\right)>0$ for all $M>0$, but this approximation has this $M_{\delta}$ cutoff. As mentioned with (1), the flux is

$$
J=h^{3} \frac{\partial p}{\partial x} .
$$

If we recognize each droplet as having a uniform value for pressure (because they are relatively locally stable), and if we let the distance between two droplets be defined as the distance between the inside edges of adjacent drops, we can rewrite $(16)$ as $[5,11]$

$$
J_{i+1, i}=h^{3} \frac{\partial p}{\partial x} \approx \frac{h^{3}\left(p_{i+1}-p_{i}\right)}{\left(X_{i+1}-W_{i+1}\right)-\left(X_{i}+W_{i}\right)}
$$

where $X_{i}$ is the position and $W_{i}$ is the width of the $i^{\text {th }}$ droplet. Using (12) and (10) and recognizing that mass flux happens via the ultra thin layer $(h=\delta)$ we can write

$$
J_{i+1, i}=\frac{k \delta^{3}\left(M_{i+1}^{-1 / 2}-M_{i}^{-1 / 2}\right)}{\left(X_{i+1}-W_{i+1}\right)-\left(X_{i}+W_{i}\right)}
$$

and $k=\left(2 A^{3} / 3\right)^{1 / 2}$ will be treated as a parameter for the influence of the disjoining pressure. Now, using (15), we can write the ODEs that govern mass exchange and movement as

$$
\frac{d M_{i}}{d t}=k \delta^{3}\left(\frac{M_{i+1}^{-1 / 2}-M_{i}^{-1 / 2}}{\left(X_{i+1}-W_{i+i}\right)-\left(X_{i}+W_{i}\right)}-\frac{M_{i}^{-1 / 2}-M_{i-1}^{-1 / 2}}{\left(X_{i}-W_{i}\right)-\left(X_{i-1}+W_{i-1}\right)}\right)
$$




$$
\frac{d X_{i}}{d t}=-\frac{B \delta^{2}}{\ln \left(\frac{M_{\delta}}{M_{i}}\right)}\left(\frac{M_{i+1}^{-1 / 2}-M_{i}^{-1 / 2}}{\left(X_{i+1}-W_{i+i}\right)-\left(X_{i}+W_{i}\right)}+\frac{M_{i}^{-1 / 2}-M_{i-1}^{-1 / 2}}{\left(X_{i}-W_{i}\right)-\left(X_{i-1}+W_{i-1}\right)}\right)
$$

where $B$ is the proportionality constant for $C_{x}$.

We will approximate solutions to initial value problems of this system of $2 N$ equations for $N$ droplets numerically using the ode45 solver via MATLAB. The initial conditions refer to the initial masses $M_{i}$ and positions $X_{i}$ of the droplet array for $i=1,2, \ldots, N$. Also, the system will depend on which type of boundary conditions we choose to use. One type of boundary condition is unbounded, so the droplets are free to move along a domain that is infinitely long, and because no droplet leaves the domain, there is no flux at the edges. Although this is not necessarily what occurs physically, it can be considered a good approximation in the case that each droplet is very small when compared to the total length of the domain. The other type of boundary condition we use is a periodic boundary condition, where the left edge and right edge are essentially connected. The leftmost and rightmost drop will be adjacent, or, if $X_{l}$ and $X_{r}$ are the positions of the leftmost and rightmost drops respectively, the distance between their drop edges is $\left(X_{l}-W_{l}\right)+\left(L-\left(X_{r}+W_{r}\right)\right)$. Physically, a periodic domain can be thought as a circle with circumference $L$.

These boundary conditions can be compared to the boundary condition of a simple finite domain where no flux of fluid leaves through the boundaries. An equivalent manner in which to imagine this condition is to create reflected drops across $x=0$ and across $x=L$. In other words, the leftmost drop will be influenced by an equal sized drop that lies to its left and the rightmost drop will be influenced by at equal sized drop that lies to its right. In our simulations, this means we create two auxiliary drops, 0 and $n+1$ such that $M_{n+1}=M_{n}$ and $M_{0}=M_{1}$. Thus, from (18), $J_{1,0}=J_{n+1, n}=0$. Therefore we have no flux at the boundaries on a simple $0 \leq x \leq L$ domain.

\subsection{Droplet Singularity Events}

Mathematically, the equations (19) remain defined and valid as long as the right-hand sides are uniformly bounded. This condition holds (and the flux will not blow up) provided that the solution does not produce a finite time singularity, which happens if

(i) $\left(X_{i+1}-W_{i+i}\right)-\left(X_{i}+W_{i}\right) \rightarrow 0 \quad$ or

(ii) $M_{i} \rightarrow M_{\delta}=M_{c}$

for any $i$ in the array. Physically, condition (ii) means the mass of a droplet approaches a critical minimum value $M_{c}$. Condition (i) means the distance of separation between adjacent droplets goes to zero. In (19b), $M_{\delta}$ represents the mass of a droplet that is very close to collapsing into the ultra thin layer. Therefore, we set $M_{c}=M_{\delta}$.

\subsubsection{Events and an example}

The solutions to these equations are calculated numerically until an event occurs that necessitates a change in the system. More precisely, events for our purposes are predetermined conditions such that if the numerical solutions to the differential equation 
meet one of these conditions, the solving halts, changes to the system are made, and then the numerical solving restarts. This idea describes how the system of droplets "coarsens"; when one of the conditions in (20) occurs, we halt the system, $N \rightarrow N-1$ by removing a drop that caused this condition, and the system is restarted. We call this phenomenon coarsening because it describes the film's evolution toward a smaller number of drops.

For an illustration of an event, we will look at the behavior of a simple differential equation for a single droplet collapse. If we consider the evolution of mass of a drop that is much smaller than its neighbors and whose position remains relatively constant, then (19a) is well approximated by the piecewise-defined differential equation

$$
\frac{d M}{d t}= \begin{cases}-\frac{2}{\sqrt{M}} & \text { if } M>M_{c} \\ 0 & \text { else }\end{cases}
$$

where $M_{c}=0$ will be our critical mass and the separation between drops has been normalized. The second case in (21) is written in the case that the mass is 0 . Namely if the drop has vanished its mass will remain at 0 .

If we start with an initial positive mass $M_{0}$, we use the first case of the differential equation. This has the analytic solution

$$
M=\left(-3 t+M_{0}^{2 / 3}\right)^{3 / 2}
$$

The mass will continue to get smaller at an increasing rate until $M=M_{c}$, or when

$$
t_{c}=\frac{M_{0}^{2 / 3}-M_{c}^{2 / 3}}{3}
$$

When $M_{c}=0$, the condition above is met at a critical time $t_{c}=M_{0}^{2 / 3} / 3$.

In (21), the rate at which the mass is decreasing diverges as $M \rightarrow 0$ (representing a finite time singularity). However, when this occurs, the MATLAB events option within the ode45 function recognizes that at this moment, $M=0$ and halts the solution. The second case of the differential equation then comes into effect with restart mass $M_{r}=0$. This solution is shown in figure 4 .

In (21) if we set the event function to halt when when $M=M_{c}=0$, then a few data points generated by the numerical solutions in MATLAB might overshoot this value and create computational problems. Since this is the case, we define a small positive threshold $\epsilon$ in order to halt the system before this error occurs. For this system, the difference in the mass at the halting was only off by about most $1 / 500$, so using a small threshold did not create a significant difference in the system. We estimate that the same is true when we use small thresholds for our coarsening dynamical system with many droplets and that the value difference will not significantly affect our results.

For this system and using a small positive threshold, there is also the question of how much the time at which the critical event occurs differs from the true value. We saw $t_{c}=M_{0}^{2 / 3} / 3$ so if instead we solve for when $\epsilon=\left(-3 t+M_{0}^{2 / 3}\right)^{3 / 2}$, we find that $t_{\text {threshold }}=\left(M_{0}^{2 / 3}-\epsilon^{2 / 3}\right) / 3$. Thus, the bias of the threshold time is $-\epsilon^{2 / 3} / 3$. We notice that as $\epsilon$ gets small, the error of the threshold time gets small. 


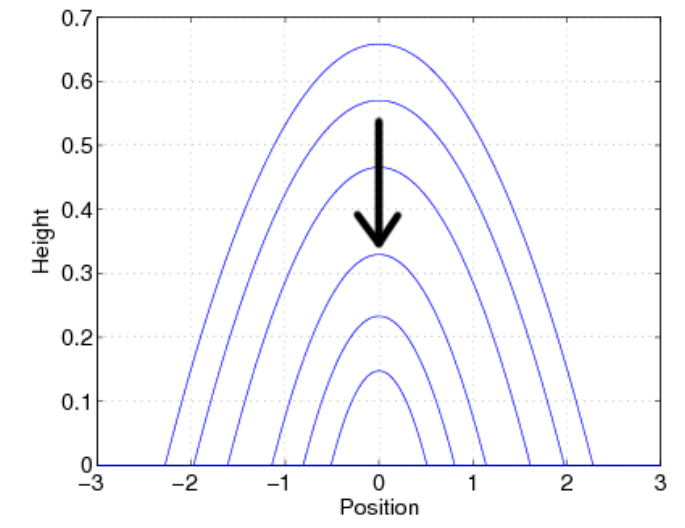

(a) Profile of a shrinking droplet at various times.

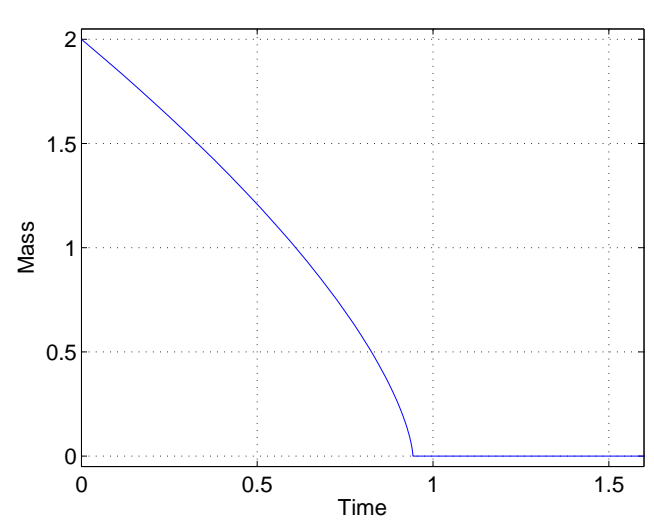

(b) Solution to (21) with initial mass $M_{0}=2$.

Figure 4: Simple one-drop system with events.

\subsubsection{Events in our system}

Numerically, a collapse event is detected if $M_{i} \leq\left(M_{\delta}+\epsilon_{1}\right)$ for some $i$ from the set of $N$ drops being described by equations (19). At that moment of time, drop $i$ is deleted from the array, and for all $1<j<N, j \neq i$, if $j<i$, drop $j$ is unchanged, and if $j>i$, drop $j$ is renumbered to become drop $j-1$. After this shifting, the numerical solving continues with $N-1$ pairs of equations.

Ideally, our system would have $\epsilon_{1}=0$ because a mass collapse should coincide with the moment when $M_{i}=M_{\delta}$. However, when $M_{i}$ gets close to this value, $\ln \left(\frac{M_{\delta}}{M_{i}}\right)$ in (19b) gets arbitrarily large. As mentioned before, the numerical solutions have finite accuracy, and it is possible that the numerical solutions might overshoot the correct answer and create problems. For this reason, we consider $\epsilon_{1}$ to be a small positive threshold so such problems do not arise. By doing this, there will be some small errors because mass will not necessarily be conserved - droplets are deleted from the system before they lose all of their mass. However these errors are small and predictable in size and will decrease when $\epsilon_{1}$ is reduced.

A similar method is used in the case that $\left(X_{i+1}-W_{i+i}\right)-\left(X_{i}+W_{i}\right) \leq \epsilon_{2}$ for some small $\epsilon_{2}>0$ (Here, $\epsilon_{2}>0$ for the same reason $\epsilon_{1}>0$ ). If this threshold is crossed for any $0<i<N$, this physically means that two adjacent droplet edges are overlapping. When this occurs, because mass is conserved, the two drops are replaced by a single drop with mass $M_{i}+M_{i+1}$. To decide the new position of the merged droplet, we imagine that when the two edges collide, the outer edges stay relatively fixed while the inside equilibrate from a two-hump drop to a one-hump drop. Once this is done, we can think of the outer edges adjusting to the new mass. For this reason, we let the new position of two merged droplets be $[5,11]$

$$
X_{i, \text { new }}=\frac{\left(X_{i+1}+W_{i+i}\right)+\left(X_{i}-W_{i}\right)}{2} .
$$


When a merging event occurs, if $j>i+1$, drop $j$ is renumbered to become drop $j-1$, and we then continue solving the system with $N-1$ paired equations. Similarly, the number of drops in the system after either of the two cases mentioned above will drop from $N$ to $N-1$. We will keep track of these numbers and denote $N(t)$ as the number of drops remaining in a particular simulation at any given time $t$ where $N_{0}=N(0)$.

\section{A First Look at Simulations}

Figure 5, which plots the droplet paths over time of 20 initial droplets, helps give intuition on how these systems coarsen and depend on system parameter values. In figure 5 (a), the $B$ parameter is lower and consequently about 75 percent of the events are collapses. On the other hand, in figure $5(\mathrm{~b})$, the $B$ parameter is higher and consequently only about 25 percent of the events are collapses. We will study this phenomenon in depth in the next section.

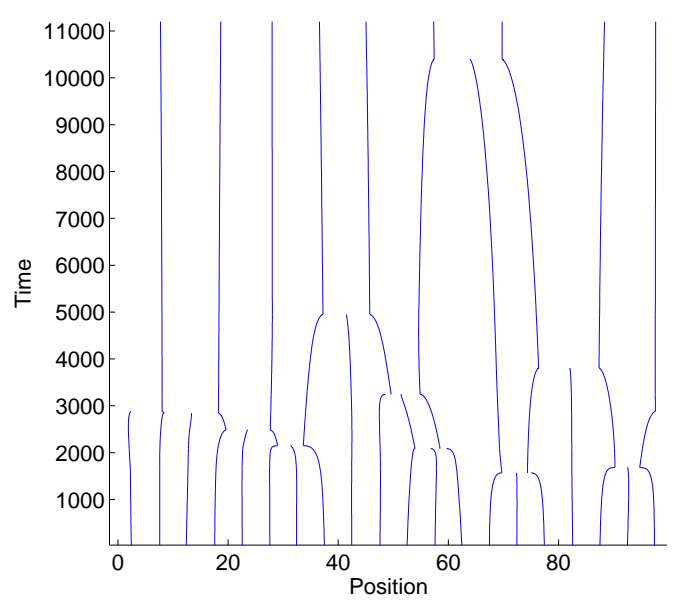

(a) Droplet paths for simulation with $N_{0}=20$ and periodic boundary conditions with $k=$ $1, B=5.5$.

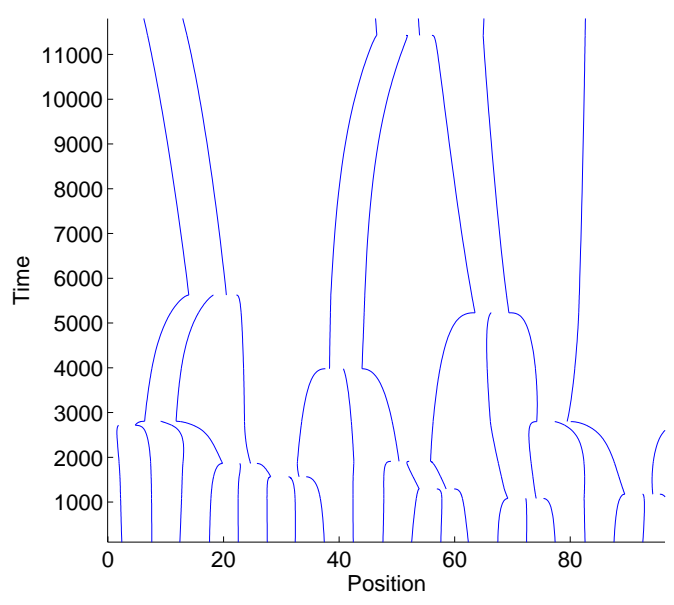

(b) Droplet paths for simulation with same 20 droplets and periodic boundary conditions with $k=1, B=11$.

Figure 5: Droplet paths over time with different parameter values.

\subsection{Evolution of number of droplets}

Over time the number of drops in the simulation will continue to decrease. One question that immediately arises is how quickly this action will happen. To get an idea, we plot the number of drops versus time for a simulation with 1000 initial drops with average mass 1 and average separation distance 5 with periodic boundary conditions. In particular, the $i^{\text {th }}$ drop has initial mass $M_{i}=1+0.2 \alpha_{i}$ and position $X_{i}=5 i+0.1 \beta_{i}$ where $\alpha_{i}$ and $\beta_{i}$ are uniform random variables on the range $[-1,1]$ that are different for each simulation. 


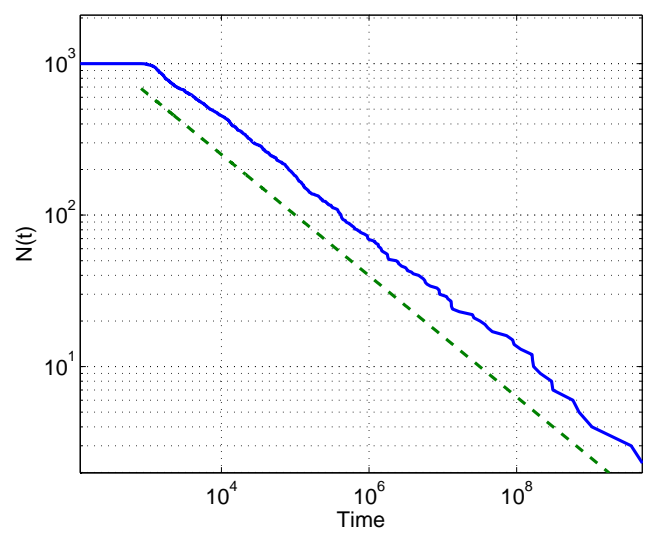

(a) Number of drops left over time in simulation with $N_{0}=1000$ and periodic boundary conditions versus $N(t) \propto t^{-2 / 5}$.

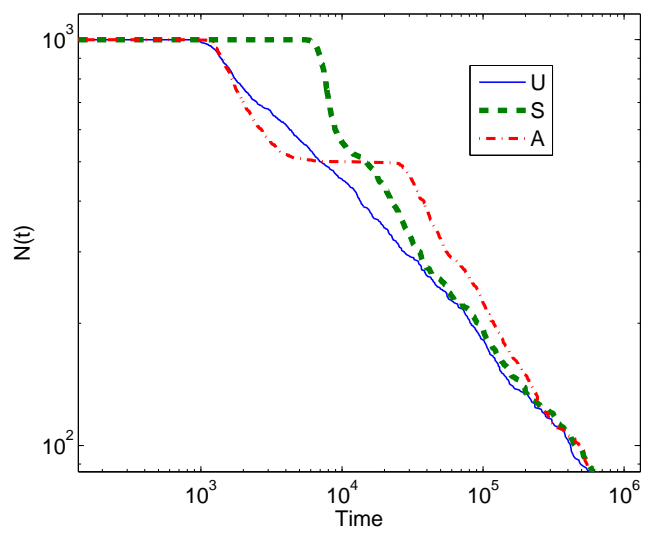

(b) Number of drops left in simulation with same 1000 drops (About first 900 drops). U-Unsorted drops (solid line). S-Linearly sorted by mass (dashed line). A-Alternatingly sorted-large differences between adjacent drops (dotted-dashed line).

Figure 6: Number of drops over time.

Figure $6(\mathrm{a})$, the plot of time versus $N(t)$ for this simulation, shows that over time $N(t)$ seems to trend toward some curve proportional to $t^{-2 / 5}$ as found in $[5,10,11]$.

It is informative to examine is what might change if the initial conditions are modified. In figure $6(\mathrm{~b})$ we simulate three different initial arrangements of the same drops. The solid curve "U" corresponds to the simulation with the original unsorted randomly generated drops. This curve seems to quickly approach a $-2 / 5$ power law after some time (as seen in figure 6(a)). The dashed curve "S" represents the number of drops remaining in a simulation when the same drops are arranged from smallest to largest before the solving begins. The drops perish at close to the same rate eventually. However, initially the sorted drops' behavior is different. In particular, there is a delay before it starts to match up with the unsorted simulation.

Lastly, the dotted-dashed curve "A" represents a simulation in which the sorting aims to create large differences in mass between adjacent droplets (we will call it alternating sorting). To describe the sorting pattern we first label droplets 1 to $N$, with 1 being the smallest drop and $N_{0}$ being the largest drop. The sorting then follows the pattern: $N_{0}, 1, N_{0}-2,3, N_{0}-4,5, \ldots, 6, N_{0}-5,4, N_{0}-3,2, N_{0}-1$. The simulation, as seen in figure 6(b) shows that relative to the other initial sortings, this sorting causes drops to perish more quickly at the start. However, soon after this initial decrease, the simulation experiences a long delay before the number of droplets starts to fall again. Eventually, however, this simulation approaches the $-2 / 5$ power law just like the other two simulations.

For the linearly sorted droplets, one reason for this delay is that, because the drops are sorted, they are adjacent to drops with similar mass. From (19) we see that 
smaller differences in the masses of adjacent drops yield smaller fluxes and may tend to slow down both the mass and position differential equations - the numerators will be smaller. Once many events happen, however, it is likely that the simulation has become sufficiently randomized, and will then become uncorrelated with the influence of the initial ordering.

For the alternating sorting, we see a relatively fast decrease in number of droplets. Here, the differences in drop masses is large between neighboring drops that are located at the edges of the domain. From (19) once again, large differences tend to speed up the dynamics of the system and the small drops quickly perish. However, after these small droplets are gone, the remaining droplets are large and have similar masses. The similarity in mass, like in the simulation with linear sorting, slows down the system, which creates the plateau effect as seen in figure 6(b). Eventually, however, the system sufficiently randomizes the droplets enough to again become uncorrelated with the initial ordering.

\section{Collapse Ratio}

When a drop vanishes into the ultra thin layer, we will call such an event a mass or collapse event and denote $\mu(t)$ as the number of mass events that have occurred in a simulation up to time $t$. Otherwise, if the second type of event occurs, we will call it a collision event and denote $\sigma(t)$ as the number of droplet collisions that have occurred up to time $t$. Clearly at any time the original total number of drops can be partitioned into $N_{0}=\mu(t)+\sigma(t)+N(t)$. We will denote the "collapse ratio" as $R(t)=\frac{\mu(t)}{\mu(t)+\sigma(t)}$ and examine this quantity under different conditions.

One question that arises about the collapse ratio is whether there exists time intervals during a drop simulation during which the the collapse ratio differs noticeably from the collapse ratio over any other interval or over the entire simulation. Generally it was the case that, in fact, the ratio varied greatly during many different intervals. When we examine the mass events and the collision events over time, as seen in Figure 7(a) which was a run with 1000 drops with periodic boundary conditions, we see that collisions appear to level off over time, while collapses continue to occur throughout the entire droplet simulation.

\subsection{Conjecture on the collapse ratio}

One possible explanation for this system's favoring of collapses later is the tendency for the average distance between drops to increase, leading to less of a chance that drops will collide. To gain intuition on this, we assume that we can estimate all drops having equal mass $M$ with drop centers differing by length $L$ (This is never exactly the case, but should be a good approximation in an average sense). Then, using equation (13), the average distance between the drops is $L-2 \sqrt{3 M / 2 A}$. If we imagine however that half of the drops are gone, and drops are equally spaced and have equal mass, then, because mass is conserved, each drop has mass $2 M$. However, each drop now has separation $2 L-2 \sqrt{3 M / A}$, or the separation has increased by $L-(\sqrt{2}-1) \sqrt{3 M / 2 A}$. This increase in separation will make collisions less likely. As drops become larger, the 
$\ln \left(\frac{M_{\delta}}{M_{i}}\right)$ term in equation (19b) becomes larger in absolute value and thus slows down the movement of drops, contributing to collisions being less likely. Thus, we believe that this increase in distance and slow-effect outweigh the time needs for the larger droplet to collapse, causing the trend that the graph shows.

\subsection{Time-Independent Intervals}

Figure 7(b) shows the collapse ratio during each of 9 intervals of the drop simulation where the 1 st is the first 10 percent of events, the 2 nd is the next 10 percent of events, and so on. This is the average over 10 runs with $k=1$ and $B=5$ in (19) and with open boundary conditions. These graphs were generated for multiple values of $k$ and $B$. However, they were nearly identical if $k$ and $B$ varied between two different sets of simulations, but $k / B$ was the same. Thus, from this data, it appears that the collapse ratio depends only on $k / B$ and not on $k$ and $B$ independently. This can be understood by rescaling time as $t=\tilde{t} / B$; this would normalize the coefficient in (19b) and give $k / B$ as the rate coefficient in (19a).

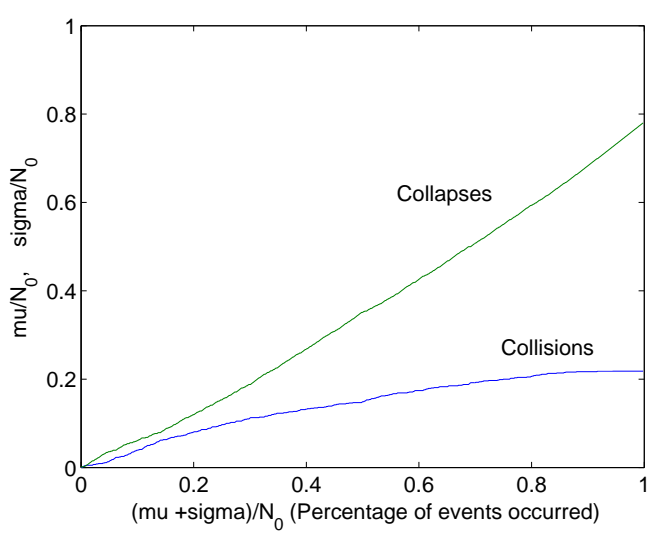

(a) Collapse and collision percentage versus percentage of events occurred.

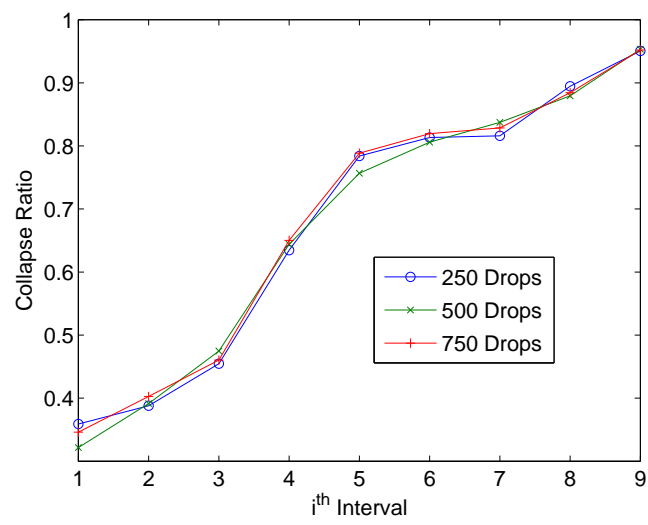

(b) Collapse ratio average of 10 simulations during the $i^{\text {th }}$ interval, where each interval has an equal number of events. $N_{0}=250,500,750$.

Figure 7: Collapse ratio over different portions of simulations.

One thing to keep in mind is that even if the number of drops in a simulation changed, the average collapse ratio looks to stay unchanged during each interval. Because the choice of 10 percent was arbitrary, we cannot extrapolate too much information from the graphs of Figure 7(b), but we certainly observe a general upward trend that appears to be $N_{0}$-independent provided that distributions of initial mass and drop distances have the same mean and variance. 


\subsubsection{Moving average}

Another way to view this increasing tendency of collapses during a simulation is to use a moving average. This uses a sliding window that recognizes a certain number of events and takes the ratio of the events in this window. In figure 8(a) we use data from a simulation with $N_{0}=1200, k / B=1 / 5$ and periodic boundary conditions to create a moving average with a time window containing 200 events. Here, we see that there

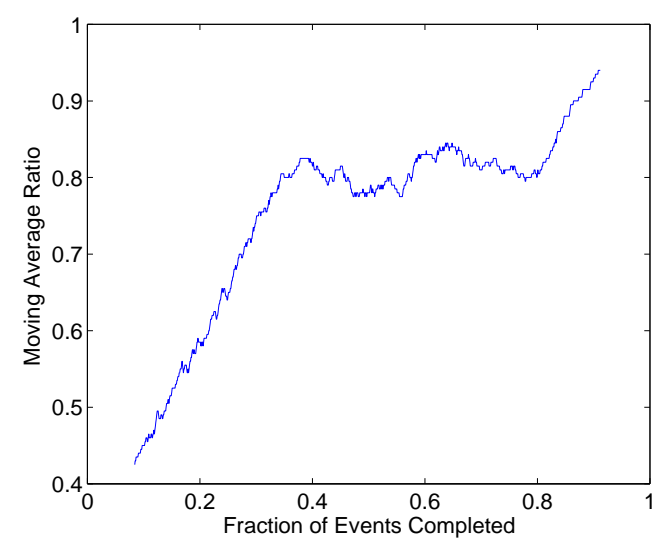

Figure 8: Collapse ratio-moving average with window of $200, N_{0}=1200$, with $k / B=1 / 5$.

is still clearly an overall upward trend. However, it does not appear to be increasing at a constant slope. We hypothesize that the cause of this lies in the distribution of the droplet masses throughout the simulation-It is not likely that after half of the the droplet events, the drop masses are uniformly distributed as they were at the beginning via initial conditions. We believe it is this phenomenon that creates the non-uniform increase.

\subsection{Collapse Ratio Over Entire Simulation}

For various values of $k$ and $B$, the overall collapse ratio was examined for multiple droplet simulations with different initial conditions and boundary conditions. We found, similar to our finding earlier, that this collapse ratio did not depend on the values of $k$ and $B$, but only on the value of $k / B$. In particular, each circle of Figure 9(a) represents the average collapse ratio of 10 simulations each with $N_{0}=250$ and initial conditions of $M_{i}=1+0.1 \alpha_{i}$ and $X_{i}=5 i+0.1 \beta_{i}$ where $\alpha_{i}$ and $\beta_{i}$ are uniform random variables on $[-1,1]$ that are different for each simulation. This was created with unbounded boundary conditions (an infinitely-long thin layer left of drop 1 and right of drop $N$ ) although the same pattern was found for periodic boundary conditions. From this graph, it appears that the $k / B$ ratio is a strong predictor of the simulation's collapse ratio. 


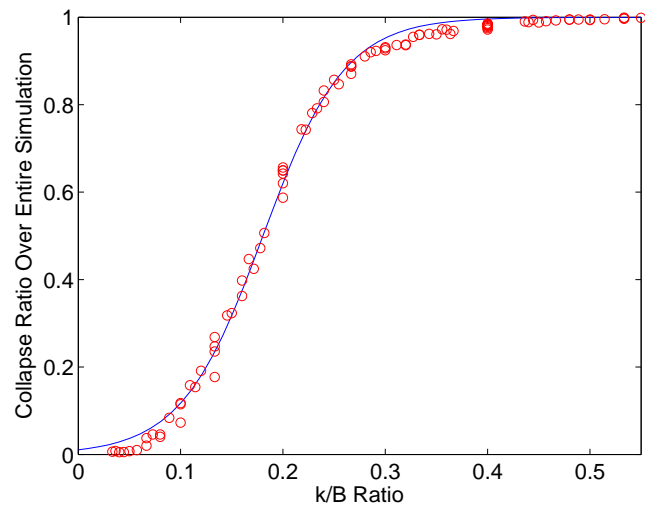

(a) Logistic function fitted to data. Each data point corresponds to the average of 10 runs with $N_{0}=250$.

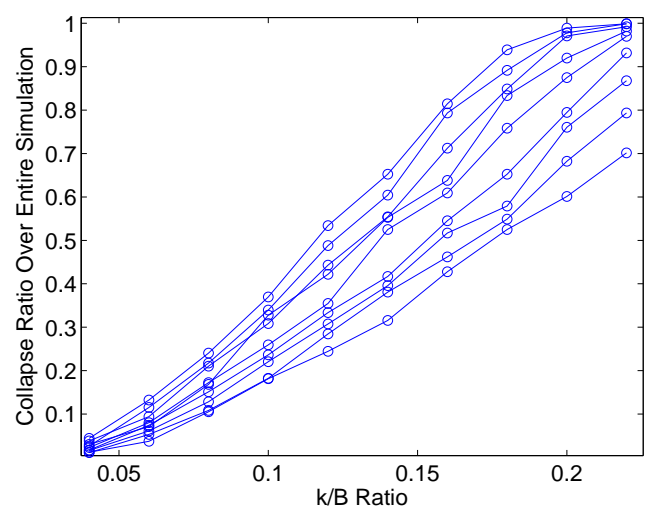

(b) Collapse Ratio with different average initial drop separation distances.

Figure 9: Collapse Ratio Graphs-Over Entire Simulation.

In figure $9(\mathrm{a})$, we use a variation of the logistic function

$$
F(x)=\frac{e^{\beta x}}{e^{\beta x}+\alpha}
$$

as a fit for the data. Here $\beta=25.09$ and $\alpha=92.48$ and we can think of the overall collapse ratio $R$ in Figure 9 (a) as $R=R\left(t_{f}\right)$ where $t_{f}$ is a time after which nearly all drops have perished. Although this variant of the logistic function fits very well to this graph, it is not obvious why this is the case, other than the fact that both the function and the data have range $[0,1]$. For now, we will leave as an open question for future work if there is a mathematical reason for why this function gives a good fit.

Figure 9 (b) shows how the data change when the average distance between each drop increases. Here, each data point is a single drop simulation with 1000 drops with average mass 1.1 and average separation length ranging from 5 to 6.6 with increments of 0.2 . The right most curve has average distance 5 while the curves further to the left represent greater drop separation distances. Clearly as the average distance between drops increases, collisions become less likely which is what we expected (it is harder to collide when drops are farther apart). Observing this, an interesting question is if the collapse ratios over a simulation can be simplified into one curve by using some scaling for the horizontal axis in the form $M_{s}^{\gamma} L_{s}^{\zeta}(k / B)$ for some scaling constants $\gamma$ and $\zeta$ where $M_{s}$ is the average initial mass and where $L_{s}$ is the average initial separation distance.

\subsection{Scaling}

For different $k / B$ ratios, we run drop simulations with 1000 drops each with initial average mass ranging from 0.5 to 1.5 in increments of 0.2 and with initial average drop separation distance ranging from 5.0 to 6.6 in increments of 0.2. In figure 10(a), we see 
that if we scale the horizontal axis as $(k / B) L_{s}^{4 / 3}$, then the six different initial average masses each seem to lie on separate curves. Moreover, we see in figure 10(b) that if we scale the horizontal axis as $M_{s}^{-1} L_{s}^{4 / 3}(k / B)$ then all 540 simulations collapse onto a single curve.

The fact that these simulations can be made to lie on a single curve despite differing initial conditions is very essential to the simplification of the thin-film dynamics problem. The high number of parameters and initial conditions within the problem creates enormous complexity. However, if we can understand something like the collapse ratio in terms of a function that is just determined by initial conditions and parameters, we can greatly reduce the difficulty of the problem for further work.

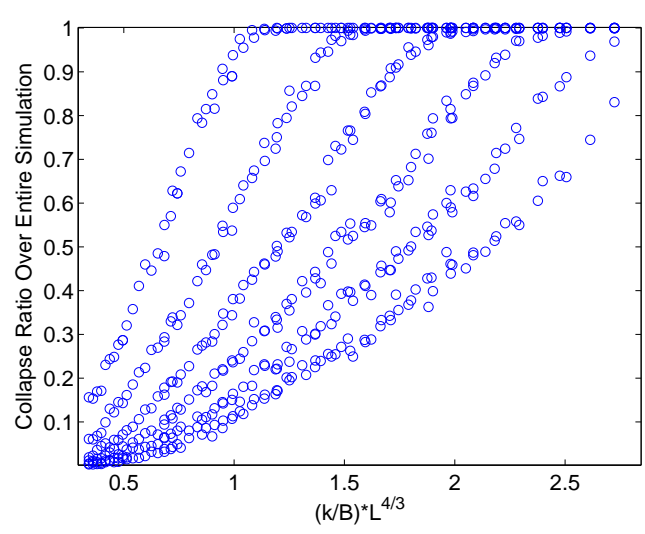

(a) Collapse Ratio graph scaled by initial average separation distance.

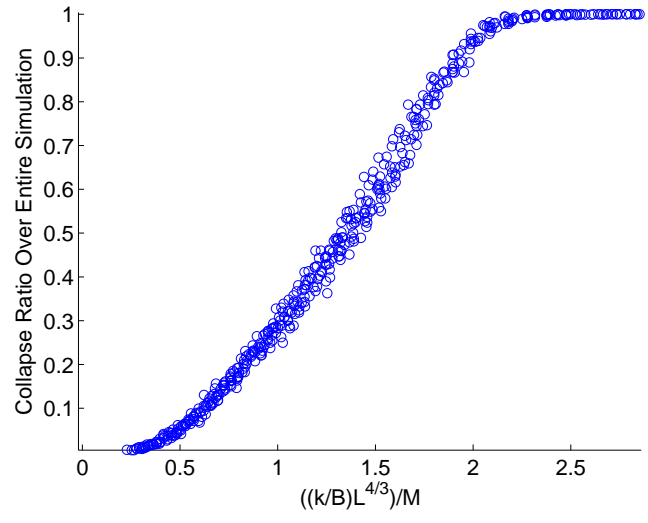

(b) Collapse Ratio graph scaled by both initial average separation distance and mass.

Figure 10: Scaled Collapse Ratio.

\section{Conclusion}

In the general sense, understanding the behavior of fluids through the use of the NavierStokes partial differential equations is extremely complicated. By using simplified and workable models of the coarsening dynamical system of thin films, we have gained some insight on some of their important properties in the context of this problem. Nonetheless, even the simplified models have presented many more questions that we have yet to answer. For example, we have observed that the $k / B$ is all that matters (not $k$ or $B$ ) when looking at quantities like the collapse ratio. Likewise, we have a good guess at what causes the trending towards more collapses over collisions throughout a simulation but we have not been precise with this. In particular, the complicated behavior in figure 8 suggests that the droplet distribution may be more of a factor in determining the collapse ratio than we expected. Although many questions still remain unanswered about this system, we believe that the reductions we have made and the ideas we have expressed will allow future research on the subject to gain an even better understanding of these systems. 


\section{Acknowledgments}

I would like to thank Thomas Witelski for advising me throughout this project and the NSF grant 0239125 for financial support.

\section{References}

[1] D.J. Acheson. Elementary Fluid Dynamics. Oxford University Press, 1990.

[2] J. Becker, G. Grun, R. Seemann, H. Mantz, K. Jacobs, K. R. Mecke, and R. Blossey. Complex dewetting scenarios captured by thin-film models. Nature Materials, 2:59-63, 2002.

[3] R. V. Craster and O. K. Matar. Dynamics and stability of thin liquid films. Rev. Mod. Phys., 81:1131, 2009.

[4] P. de Gennes, F. Brochard-Wyart, and D. Quere. Capillarity and Wetting Phenomena: Drops, Bubbles, Pearls, Waves. Springer, 2004.

[5] M. B. Gratton and T. P. Witelski. Transient and self-similar dynamics in thim film coarsening. Physica D, 238(23-24):2380-2394, 2009.

[6] K. Jacobs, R. Seemann, and S. Herminghaus. Stability and dewetting of thin liquid films. In Thin Liquid flims. World Scientific, 2008.

[7] L. Kondic. Instabilities in gravity driven flow of thin fluid films. SIAM Rev., 45(1):95-115, 2003.

[8] T. G. Myers. Thin films with high surface tension. SIAM Review, 40(3):441-462, 1998.

[9] A. Oron, S. H. Davis, and S. G. Bankoff. Long-scale evolution of thin liquid films. Reviews of Modern Physics, 69:931-980, 1997.

[10] T.P. Witelski and K.B. Glasner. Coarsening dynamics of dewetting films. Physical Review E, 67:016302, 2003.

[11] T.P. Witelski and K.B. Glasner. Collision versus collapse of droplets in coarsening of dewetting thin films. Physica D, 209:80-104, 2005. 